University of Nebraska - Lincoln

DigitalCommons@University of Nebraska - Lincoln

May 2003

\title{
Spin structure at nanojunctions and constrictions
}

\author{
Ralph Skomski \\ University of Nebraska-Lincoln, rskomski2@unl.edu \\ David J. Sellmyer \\ University of Nebraska-Lincoln, dsellmyer@unl.edu
}

Follow this and additional works at: https://digitalcommons.unl.edu/physicssellmyer

Part of the Physics Commons

Skomski, Ralph and Sellmyer, David J., "Spin structure at nanojunctions and constrictions" (2003). David Sellmyer Publications. 38.

https://digitalcommons.unl.edu/physicssellmyer/38

This Article is brought to you for free and open access by the Research Papers in Physics and Astronomy at DigitalCommons@University of Nebraska - Lincoln. It has been accepted for inclusion in David Sellmyer Publications by an authorized administrator of DigitalCommons@University of Nebraska - Lincoln. 


\title{
Spin structure at nanojunctions and constrictions
}

\author{
R. Skomski ${ }^{\mathrm{a})}$ and D. J. Sellmyer \\ Department of Physics and Astronomy and Center for Materials Research and Analysis, \\ University of Nebraska, Lincoln, Nebraska 68588
}

(Presented on 14 November 2002)

\begin{abstract}
A micromagnetic Green-function approach is used to investigate the effect of nanojunctions, constraints, and other obstacles on spin-dependent conduction. Depending on geometry, the determination of the spin structure involves several types of Bessel functions. A common feature of the Green functions is the involvement of the domain-wall width of the main phase, which can be interpreted as the decay length of the magnetization perturbation away from the junction. This length is typically on the order of $10 \mathrm{~nm}$ and independent of the strength of the perturbation. Only the magnitude of the magnetization perturbation depends on the strength of the inhomogenity. A particular feature of the considered structures is that the total spin-dependent scattering cross section, as estimated from the squared magnetization gradient, exhibits a characteristic real-structure dependent maximum as a function of the boundary phase or junction dimensions. (C) 2003 American Institute of Physics. [DOI: 10.1063/1.1558666]
\end{abstract}

\section{INTRODUCTION}

Magnetic nanojunctions and other inhomogeneities, such as granular interfaces, are of considerable interest in the context of spin electronics. ${ }^{1-7}$ Since the scattering of conduction electrons depends on the magnetization gradient $\nabla \mathbf{M}(\mathbf{r})$, the determination of the micromagnetic spin structure is of great importance. ${ }^{8,9}$ In the past, theoretical research has largely focused on quasione-dimensional structures, which have been tackled by phenomenological continuum and atomicresolution methods. ${ }^{10-14}$ From a theoretical point of view, the spin structure at granular interfaces and in constrained domain walls was first investigated in the context of polycrystalline rare-earth transition-metal intermetallics, ${ }^{10}$ although various earlier articles, such as Refs. 15-19, anticipate much of the involved physics. A key feature is magnetization and energy-density tails extending well into the bulk of the grains. In hard magnets, the corresponding penetration length is proportional to the wall-width parameter $\delta_{\mathrm{o}}=\sqrt{A / K_{1}}$, whereas in soft magnets it is $l_{\mathrm{o}}=\sqrt{A / \mu_{\mathrm{o}} M_{s}^{2}}$. ${ }^{20}$ Both lengths are typically on the order of a few nanometers.

At granular interfaces, both reduced grain-boundary exchange and grain misalignment contribute to the perturbation of the spin structure and define an effective intergranular exchange. ${ }^{13,14}$ Furthermore, the reduced exchange at interfaces gives rise to a quasidiscontinuity of the magnetization. In the absence of external fields it has a relative strength of $1 /\left(1+2 A^{\prime} \delta_{0} / A D\right)$, where $D$ is the thickness of the interface, and $A$ and $A^{\prime}$ are the exchange stiffnesses in the bulk and in the interface region, respectively. ${ }^{14}$ A detailed comparison of continuum and atomically resolved models yields only minor corrections due to the discrete nature of the layerresolved model. ${ }^{13,14}$

a) Author to whom correspondence should be addressed; electronic mail: rvdskomski@msn.com
A common feature of the above-mentioned structures is their one-dimensional nature, but many geometries of interest in spin electronics are quite complicated and cannot be described in terms of planar models. One key aspect of this article is to discuss three-dimensional effects.

\section{BOUNDARY CONDITIONS}

The local magnetization $\mathbf{M}(\mathbf{r})$ is obtained by finding and analyzing the local minima of the free-energy functional

$$
\begin{aligned}
F= & \int\left\{A\left[\nabla\left(\frac{\mathbf{M}}{M_{s}}\right)\right]^{2}-K_{1} \frac{(\mathbf{n} \cdot \mathbf{M})^{2}}{M_{s}^{2}}-\mu_{\mathrm{o}} \mathbf{M} \cdot \mathbf{H}\right. \\
& \left.-\frac{\mu_{\mathrm{o}}}{2} \mathbf{M} \cdot \mathbf{H}_{d}(\mathbf{M})\right\} d V .
\end{aligned}
$$

Here $M_{s}(\mathbf{r})$ is the spontaneous magnetization, $K_{1}(\mathbf{r})$ is the first uniaxial anisotropy constant, $A(\mathbf{r})$ denotes the exchange stiffness, and $\mathbf{n}(\mathbf{r})$ is the unit vector of the local anisotropy direction. $\mathbf{H}$ is the external magnetic field, and $\mathbf{H}_{d}$ is the magnetostatic self-interaction field. All parameters entering Eq. (1) are local parameters, because they depend on chemistry, crystal structure, and crystallite orientation. For example, the anisotropy $K_{1}(\mathbf{r}, T)$ is easily changed by varying the chemical composition. ${ }^{20}$

For simplicity, we restrict ourselves to linear case, which is realized, e.g., in weakly textured systems. Nonlinear corrections yield, for example, a wall-width enhancement by a factor of $\pi / 2$, but the essential physics remains unchanged. We start by rewriting the easy axis direction as $\mathbf{n}(\mathbf{r})$ $=\sqrt{1-a^{2}(\mathbf{r})} \mathbf{e}_{z}+\mathbf{a}(\mathbf{r})$, where $\mathbf{a}(\mathbf{r})$ are the transverse vector components of $\mathbf{n}$. A similar equation exists for M. Series expansion yields $\mathbf{n}=\left(1-a^{2} / 2\right) \quad \mathbf{e}_{z}+\mathbf{a}$ and $\mathbf{M}=M_{s}(1$ $\left.-m^{2} / 2\right) \mathbf{e}_{z}+M_{s} \mathbf{m}$. Next we assume that $\mathbf{H}=H \mathbf{e}_{z}$, so that putting $\mathbf{M}$ and $\mathbf{n}$ into Eq. (1) leads to

$$
E=\int\left[A(\nabla \mathbf{m})^{2}+K_{1}(\mathbf{m}-\mathbf{a})^{2}+\frac{1}{2} \mu_{\mathrm{o}} M_{s} H \mathbf{m}^{2}\right] d \mathbf{r} .
$$




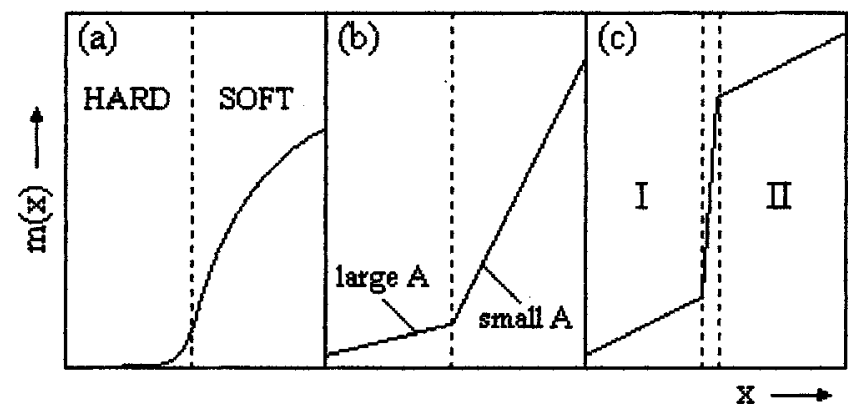

FIG. 1. Boundary conditions and exchange stiffness $A$ : (a) hard-soft interface with common $A$, (b) interface between two ferromagnetic phases with different $A$, and (c) quasidiscontinuity due to reduced $A$ in the grain boundary.

Here we have ignored a physically unimportant zero-point energy and, in fair approximation, ${ }^{21}$ incorporated the magnetostatic self-interaction into $K_{1}$ and $H$. To minimize $E$ with respect to $\mathbf{m}(\mathbf{r})$ we exploit that minimum of any functional $F=\int \eta d V$ is given by the functional derivative $\delta F / \delta \mathbf{m}(\mathbf{r})$ $=0$. Explicitly,

$$
\frac{\delta F}{\delta \mathbf{m}(\mathbf{r})}=-\nabla\left(\frac{\partial \eta}{\partial \nabla \mathbf{m}(\mathbf{r})}\right)+\frac{\partial \eta}{\partial \mathbf{m}(\mathbf{r})}
$$

so that ${ }^{10}$

$$
-\nabla(A \nabla \mathbf{m})+\left(K_{1}+\frac{1}{2} M_{s} H\right) \mathbf{m}=K_{1} \mathbf{a}(\mathbf{r}) .
$$

This equation means that the polycrystalline easy-axis disorder $\mathbf{a}(\mathbf{r})$ acts as an inhomogeneity.

The term $\nabla(A \nabla \mathbf{m})$ in Eq. (4) reflects the local character of the exchange stiffness $A(\mathbf{r}){ }^{19}$ For sharp phase boundaries, the exchange term reduces to the boundary condition

$$
\left.\left(A(x) \frac{\partial m}{\partial x}\right)\right|_{x_{\mathrm{O}^{-\varepsilon}}}=\left.\left(A(x) \frac{\partial m}{\partial x}\right)\right|_{x_{\mathrm{O}^{+\varepsilon}}} .
$$

Figure 1 illustrates the physical meaning of this boundary condition. A jump in $A(x)$ yields a change in the slope of the perpendicular magnetization component $m(x)$. Reduced grain-boundary exchange yields the above-mentioned quasidiscontinuity of the magnetization, which is unrelated to the hard or soft character of the involved phases.

\section{NANOJUNCTIONS}

In the one-dimensional case, the solutions of Eq. (4) are piecewise exponential, proportional to $\exp \left( \pm x / \delta_{0}(x)\right)$. In the case of arbitrary geometries, it is convenient to rewrite Eq. (4) as a linear operator equation, $\mathbf{Q m}=\mathbf{a}$. The formal solution of this equation is $\mathbf{m}=\mathbf{G a}$, or, in real space, $\mathbf{m}(\mathbf{r})$ $=\int \mathbf{G}\left(\left|\mathbf{r}-\mathbf{r}^{\prime}\right|\right) \mathbf{a}\left(\mathbf{r}^{\prime}\right) d V^{\prime}$, where $\mathbf{G}=\mathbf{Q}^{-1}$ is the propagator of the micromagnetic problem (micromagnetic Green function). ${ }^{10} G(r)$ is proportional to $K_{d / 2-1}\left(r / r_{\delta}\right)$, where $K_{m}$ is Macdonald's modified Bessel function on the order of $m$ and $r_{\delta}$ is the interaction length of the problem. ${ }^{22}$ In the absence of strong magnetic fields, $r_{\delta} \approx \delta_{\mathrm{o}}=\sqrt{A / K_{1}}$. Typical $\delta_{\mathrm{o}}$ values are $12.9,4.4$, and $26.1 \mathrm{~nm}$ for $\mathrm{Fe}, \mathrm{Co}$, and $\mathrm{Ni},{ }^{20}$ corresponding to 51,17 , and 107 interatomic distances, respectively. For one-dimensional problems, $G(r)$ is exponential, whereas three-dimensional problems are described by
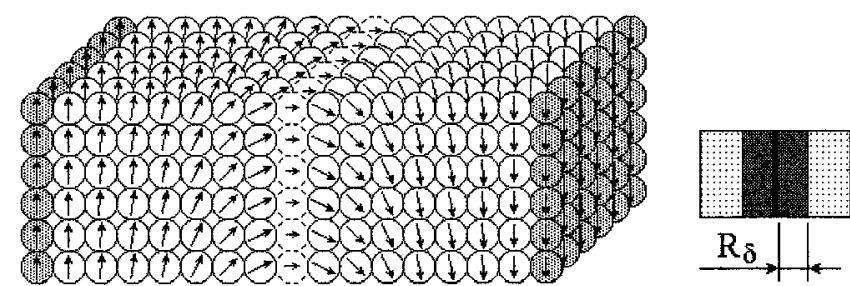

(a)
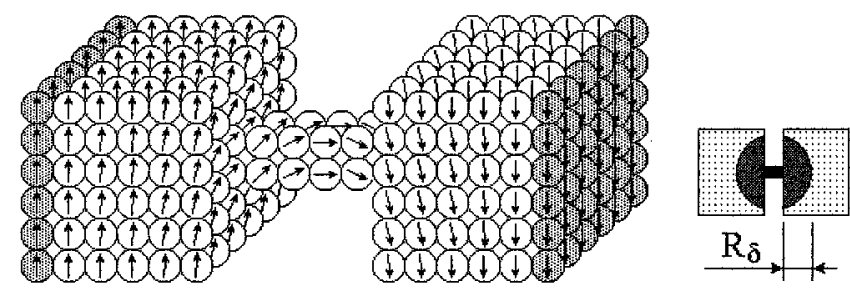

(b)

FIG. 2. Exchange effects and spin structure: (a) reduced interface exchange and (b) narrow junction.

$K_{1 / 2}\left(r / r_{\delta}\right) \sim \exp \left(-r / r_{\delta}\right) / r$. Figure 2 illustrates the meaning of this function by comparing a nanojunction with a onedimensional grain boundary.

In Fig. 2(b), the semi-infinite character of the connected ferromagnetic bodies does not affect $G(r)$ very much, because the boundary conditions discussed above imply that $\partial \mathbf{m} / \partial \mathbf{r}_{\perp}=0$ at free surfaces. Surface anisotropies lead to a partial clamping of the magnetization, although the effect is less pronounced than in Ref. 23. Note that $r_{\delta}$ does not depend on the strength of the inhomogenities. Varying the exchange in the interface region in (a) or the size or coupling strength of the junction in (b) affects the amplitude of the perturbation but leaves its range $r_{\delta}$ unchanged. This complicates the determination of the spin structure at nanojunctions and similar features from first principles, because the perturbed regions tend to contain thousands or even millions of atoms and because the involved energy differences are very small. Note that the present spin structures must not be confused with atomic-scale noncollinear structures, which involve higher energy differences and are comparatively easy to treat with first-principle calculations.

The spin-dependent scattering of electrons at nanojunctions and grain boundaries affects the magnetoresistance of spin-electronic structures. On a one-electron level, the scattering reflects the spin dependence of the exchange potential $V_{\sigma}\left(\mathbf{r}_{i}\right),{ }^{3,5,7}$ so that the resistance is a functional of the local magnetization $\mathbf{M}(\mathbf{r})$. In particular, large magnetization gradients $\nabla \mathbf{M}(\mathbf{r})$ are expected to yield strong scattering contributions. ${ }^{4}$ Typical domain walls are smooth and extend over many interatomic distances, but grain boundaries and nanojunctions have regions with very large gradients.

A crude measure to gauge the spin-dependent scattering ability of an interface is the integral $\int(\nabla \mathbf{M})^{2} d x$ $\approx M_{s}^{2} \int(\nabla \mathbf{m})^{2} d x$. This expression is maximized for interface thicknesses of the order of $D=\delta_{\mathrm{o}} A^{\prime} / A$. Most of the scattering is realized in the nanojunction. In spite of their extension, the tails contribute very little to the scattering. Compared to Bloch wall scattering, where $\int(\nabla \mathbf{m})^{2} d x$ 
$\approx 1 / \delta_{0}$, the maximum scattering is enhanced by a factor $A / A^{\prime}$. For example, taking $\delta_{0}=15 \mathrm{~nm}$ and $A^{\prime}=0.1 A$ yields a maximum scattering for boundaries having a thickness of $1.5 \mathrm{~nm}$. For thinner boundaries, the spin perturbation becomes delocalized, thereby reducing the average gradient, and in the limit of zero thickness, the quite small bulk value $1 / \delta_{0}$ is reproduced. Exchange-decoupled grains, where $A^{\prime} / A \approx 0$, would yield the largest magnetoresistance, but strong reductions of $A^{\prime}$ are likely to negatively affect the spin injection through the boundary region. Another way of enhancing the scattering is using nanojunctions, which can be shown to have a reduced optimum length $D$. For contact nanojunctions such as that shown in Fig. 2(b), the optimum length is proportional to the cross-section area of the junction. Hard materials, where $\delta_{0}$ is small, could also be used, ${ }^{13}$ but this requires large fields to switch the magnetization direction.

\section{DISCUSSION AND CONCLUSIONS}

The range of the perturbation $\delta_{\mathrm{o}}$ is a direct consequence of the functional structure of Eq. (4). However, Eq. (4) amounts a rather crude description of the magnetostatic selfinteraction in terms of shape anisotropy, which scales as $\mu_{\mathrm{o}} M_{s}^{2}$ and is incorporated into the anisotropy constant $K_{1}$. First, this ignores long-range cooperative effects such as flux-closure domains, which may occur in soft-magnetic materials. Second, in soft magnets the shape anisotropy is much larger than the magnetocrystalline anisotropy, so that the effective anisotropy is essentially equal to the shape anisotropy and the range of the perturbation is on the order of $l_{\mathrm{o}}$ $=\sqrt{A / \mu_{0} M_{s}^{2}}$. This ensures that the range of the perturbation remains finite in the soft-magnetic limit of zero magnetocrystalline anisotropy. A similar cutoff is provided by the external magnetic field, which is included in Eq. (4).

In conclusion, we have investigated how the spin structure is modified by imperfections such as grain boundaries and nanojunctions. Even for well-localized and weak imperfections the magnetization perturbation extends several nanometers into the adjacent ferromagnetic regions. For thin films and plane surfaces, the decay is described by exponential functions, whereas three-dimensional geometries lead to the involvement of modified spherical Bessel functions. Models with atomic resolution yield small corrections to the continuum results, whereas more accurate first-principle cal- culations are very difficult due to the large number of atoms and the small energies differences involved. A specific result of our calculations is that the spin-dependent scattering depends on the geometry, size, and material of the boundary or junction and exhibits a size-dependent maximum. For example, grain-boundary magnetoresistance has a maximum for some boundary thickness but approaches the very low bulk limit for very thin boundaries.

\section{ACKNOWLEDGMENTS}

This work was supported by AFOSR, ONR, ARO, and CMRA.

${ }^{1}$ A. E. Berkowitz, J. R. Mitchell, M. J. Carey, A. P. Young, S. Zhang, F. E. Spada, F. T. Parker, A. Hütten, and G. Thomas, Phys. Rev. Lett. 68, 3745 (1992).

${ }^{2}$ J. Q. Xiao, J. S. Jiang, and C. L. Chien, Phys. Rev. Lett. 68, 3749 (1992).

${ }^{3}$ Sh.-F. Zhang and P. M. Levy, J. Appl. Phys. 73, 5315 (1993).

${ }^{4}$ J. F. Gregg, W. Allen, K. Ounadjela, M. Viret, M. Hehn, S. M. Thompson, and J. M. D. Coey, Phys. Rev. Lett. 77, 1580 (1996).

${ }^{5}$ M. Viret, D. Vignoles, D. Cole, J. M. D. Coey, W. Allen, D. S. Daniel, and J. F. Gregg, Phys. Rev. B 53, 8464 (1996).

${ }^{6}$ J. J. Versluijs, M. A. Bari, and J. M. D. Coey, Phys. Rev. Lett. 87, 026601 (2001).

${ }^{7}$ Spin Electronics, edited by M. Ziese and M. J. Thornton (Springer, Berlin, 2001).

${ }^{8}$ H. D. Chopra and S. Z. Hua, Phys. Rev. B 66, 020403 (2002).

${ }^{9}$ E. Yu. Tsymbal et al., J. Phys.: Condens. Matter (in press 2002).

${ }^{10}$ R. Skomski, J. P. Liu, J. M. Meldrim, and D. J. Sellmyer, in Magnetic Anisotropy and Coercivity in Rare-Earth Transition Metal Alloys, edited by L. Schultz and K.-H. Müller (Werkstoffinformationsgesellschaft, Frankfurt, Germany, 1998), p. 277.

${ }^{11}$ P. Bruno, Phys. Rev. Lett. 83, 2425 (1999).

${ }^{12}$ K. Suzuki and J. M. Cadogan, J. Appl. Phys. 85, 4400 (1999).

${ }^{13}$ R. Skomski, in Spin Electronics, edited by M. Ziese and M. J. Thornton (Springer, Berlin, 2001), p. 204.

${ }^{14}$ R. Skomski, H. Zeng, and D. J. Sellmyer, IEEE Trans. Magn. 37, 2549 (2001).

${ }^{15}$ E. Goto, N. Hayashi, T. Miyashita, and K. Nakagawa, J. Appl. Phys. 36, 2951 (1965)

${ }^{16}$ H. R. Hilzinger and H. Kronmüller, Phys. Lett. 51A, 59 (1975).

${ }^{17}$ H. Fukunaga and H. Inoue, Jpn. J. Appl. Phys., Part 1 31, 1347 (1992).

${ }^{18}$ S. Nieber and H. Kronmüller, Phys. Status Solidi B 153, 367 (1989).

${ }^{19}$ R. Skomski and J. M. D. Coey, Phys. Rev. B 48, 15812 (1993).

${ }^{20}$ R. Skomski and J. M. D. Coey, Permanent Magnetism (Institute of Physics, Bristol, 1999).

${ }^{21}$ R. Skomski, H. Zeng, M. Zheng, and D. J. Sellmyer, Phys. Rev. B 62, 3900 (2000).

${ }^{22}$ J. Richter and R. Skomski, Phys. Status Solidi B 153, 711 (1989).

${ }^{23}$ R. Skomski, J.-P. Liu, and D. J. Sellmyer, Phys. Rev. B 60, 7359 (1999). 